\title{
Fabrication of High-Resolution, Self-Aligned Palladium Electrodes
}

Laura Vera Jennia, Johannes Weicharta, Matthias Muotha, Kiran Chikkadia, Miroslav Haluska $^{a}$ and Christofer Hierold ${ }^{\mathrm{a}}$

a Micro and Nanosystems, Department of Mechanical and Process Engineering, ETH Zürich, Zürich, Switzerland

Corresponding author: L.V. Jenni, laura.jenni@micro.mavt.ethz.ch.

Present address: Micro and Nanosystems, Department of Mechanical and Process Engineering, ETH Zürich, Zürich, Switzerland. 


\section{Abstract}

Here, a fabrication approach is presented, which allows for the patterning of vertically displaced, high density and high resolution palladium electrodes in a single processing step. A top down method, namely ion milling, was used to transfer the e-beam lithography pattern into the palladium. Hereby hydrogen silsesquioxane (HSQ) was used as a hard mask. Other than the conventionally used lift-off based approach, this method does not restrict the line height to spacing ratio and therefore permits the fabrication of very densely spaced electrodes. Specifically, electrodes with as little as $25 \mathrm{~nm}$ spacing were obtained, although only lines with spacings of $80 \mathrm{~nm}$ or more were electrically isolated without any further processing. By adjusting the tilting angle and the HSQ to Pd thickness the cross-sectional profile can be controlled and varied from triangular $\left(30^{\circ}\right.$ side-wall slope at $10^{\circ}$ tilt) to rectangular $\left(30^{\circ}\right.$ tilt). This can be used to its advantage in order to reduce the parasitic capacitance of adjacent lines for a given pitch. Additionally, a proximity effect was observed as the decrease of spacing of electrodes was correlated with an increase in side-wall verticality. In summary, we have demonstrated a robust process to pattern perfectly aligned electrodes on complex topographical substrates. The here presented method is characterized by a high degree of scalability and also high flexibility in terms of materials selection.

\section{Graphical Abstract}

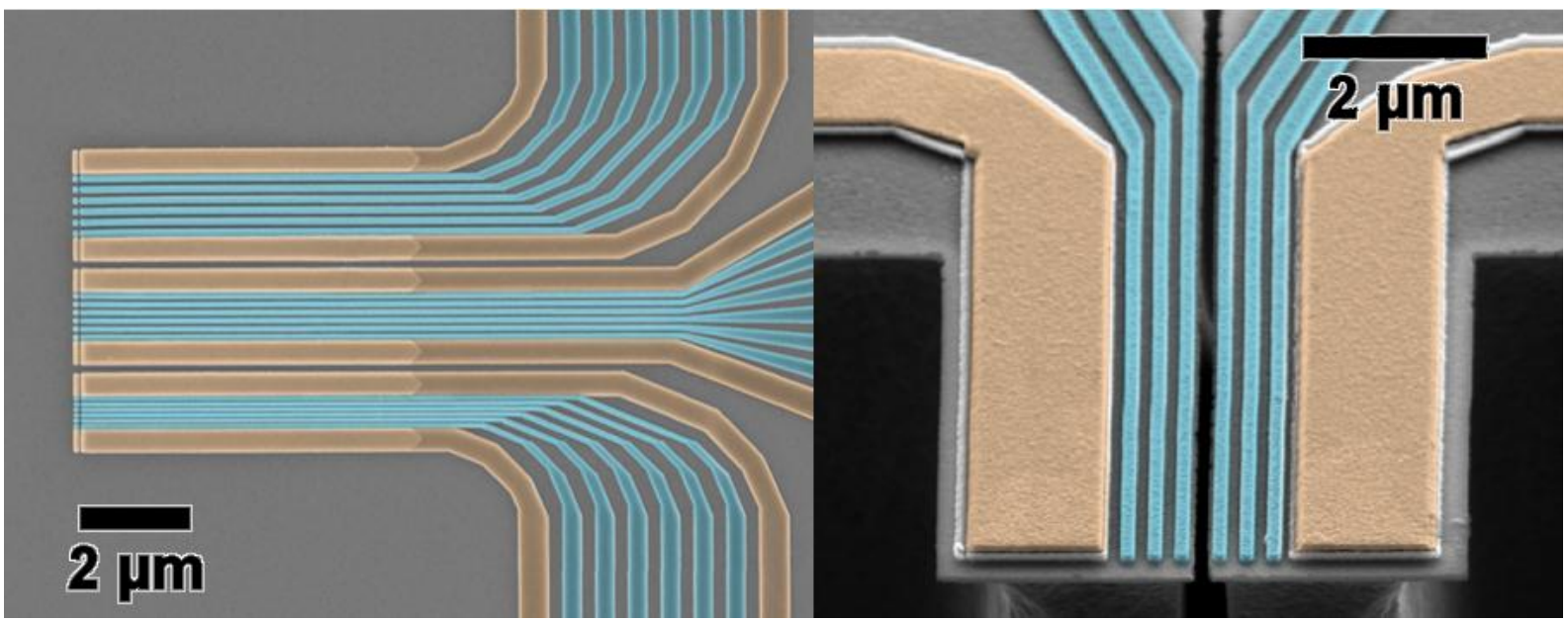

\section{Keywords}

self-aligned; palladium; ion milling; multi-gate 


\section{Introduction}

Palladium $(\mathrm{Pd})$ is known to form excellent contacts with low-dimensional carbon materials, in particular with single-walled carbon nanotubes (SWNTs). Specifically, favorable wetting properties and a high on-off current ratio have previously been observed [1]. Simultaneously palladium is difficult to chemically dry etch $[2,3]$, therefore the choice of fabrication methods for nanostructures is primarily limited to lift-off and physical etching.

Here, we present an ion milling based approach to pattern densely spaced high-resolution source, drain and gate electrodes in a single step on a prepatterned substrate. Unlike lift-off based processes this approach does not limit the spacing between adjacent electrodes with respect to the desired line height.

These vertically displaced electrodes can then later be used to transfer a carbon nanotube (CNT) from

a)

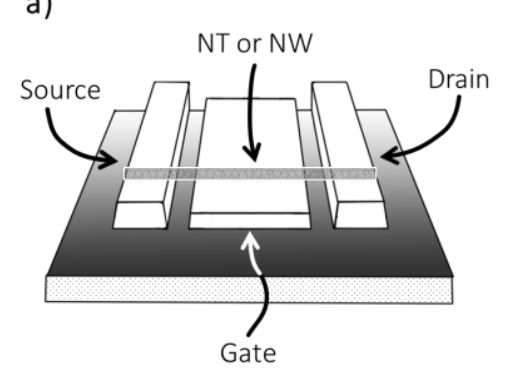

b)

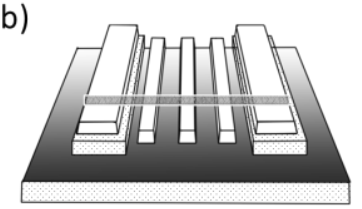

c)

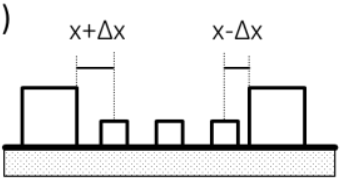

Conventional 2-step process

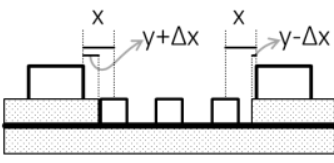

Pre-patterning of substrate a separate growth substrate on top, in order to create functional devices [4-6].

For suspended CNT devices in field-effecttransistor (FET) architecture (see Figure 1.a) scaling down the gate dimensions and electrode spacings allows for the fabrication of ultra-short channel devices, but also enables the packing of multiple gate electrodes between the source and drain (see Figure 1.b). These structures can be used for local actuation and sensing [5]. Hereby quantum-effects can be studied $[5,7,8]$ and also, in case of resonant devices, mode coupling $[9,10]$ can be investigated. Using a one-step process in comparison to a conventional 2-step process means that the exact relative location of the electrodes is well defined (see Figure 1.c). This permits a further decrease in spacing between adjacent electrodes.

d)

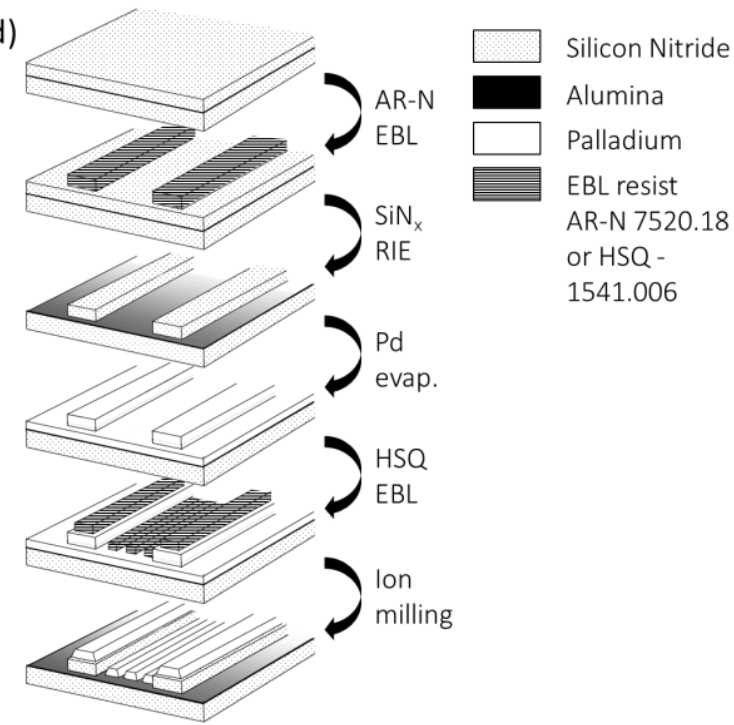

Fig. 1. a) A typical nanoresonator design. b) CNT on a multi-gate structure. c) By choosing to pattern electrodes on a pre-patterned substrate rather than using a conventional two-step process the misalignment is shifted to between the electrodes and substrate rather than the electrodes themselves. d) Fabrication steps for the here demonstrated structures.

\section{Materials and methods}

A layer stack of $200 \mathrm{~nm}$ plasma-enhanced chemical vapor deposited $\mathrm{SiN}_{\mathrm{x}}\left(\mathrm{PECVD}\right.$, at $300^{\circ} \mathrm{C}$, $120 \mathrm{sccm} \mathrm{SiH}_{4}+1000 \mathrm{sccm} \mathrm{N}$ ), $2 \mathrm{~nm}$ atomic layer deposited $\mathrm{Al}_{2} \mathrm{O}_{3}\left(\mathrm{ALD}\right.$, at $\left.250^{\circ} \mathrm{C}\right), 100 \mathrm{~nm}$ PECVD $\mathrm{SiN}_{\mathrm{x}}$ was precipitated onto a $\mathrm{Si}(100)$ wafer and annealed in a rapid thermal annealing system (AS One 150 ) at $850^{\circ} \mathrm{C}$. This results in an increased wet-etch resistance of the nitride layers [11] and the recrystallization of the alumina layer. For ALD alumina the transition to $\alpha$-alumina has been observed at temperatures as low as $750^{\circ} \mathrm{C}$ [12]. In comparison to amorphous alumina, $\alpha$-alumina is compatible to hydrofluoric acid (HF) as well as vapor HF $[3,13]$. This is required here for a release step of suspended Si-structures at a later stage. This layer stack was patterned (see Figure 1.d) 
using a negative e-beam resist (AR-N 7520.18, 400 $\mathrm{nm}$, e-beam tool: Vistec EBPG 5200+ at $100 \mathrm{kV})$. A $\mathrm{CHF}_{3}$ based RIE process was used to pattern the $\mathrm{SiN}_{\mathrm{x}}$ layer with the alumina as an etch stop.

Onto this pre-patterned substrate $2 \mathrm{~nm}$ chromium and $73 \mathrm{~nm}$ palladium were evaporated. Next, a 20 $\mathrm{nm}$ layer of $\mathrm{SiO}_{2}$ was deposited using a PECVD process $\left(300^{\circ} \mathrm{C}, 150 \mathrm{sccm} \mathrm{SiH}_{4}+710 \mathrm{sccm} \mathrm{N} 2 \mathrm{O}\right)$ to improve the surface adhesion of hydrogen silsesquioxane (HSQ, XR-1541.006), which was used as the high-resolution e-beam resist. After ebeam writing and development (with AZ351B) the layer was ion milled (Oxford lonfab 300 , ion currents of 250 to $500 \mathrm{~mA}$, acceleration voltage of $350-500 \mathrm{~V}$, at a processing pressure of $7 \cdot 10^{-5} \mathrm{Torr}$ and a platen temperature of $5^{\circ} \mathrm{C}$ ), while using secondary ion mass spectroscopy (SIMS) to detect when the end of the process was reached. Palladium is highly chemically stable. In particular in fluorine based plasmas a Pd-F film evolves, which is even more chemically stable than bulk $\mathrm{Pd}$ [2]. Chemical etching of palladium in chlorine plasmas is as of yet unreported. As a result it can only be effectively etched by sputtering [3]. HSQ was chosen for ion milling due to its low etch rate in comparison to $\mathrm{Pd}$, its high resolution and low line roughness $[3,14]$.

The structures were analyzed electrically (resistance and leakage current measurements) and by scanning electron microscopy (SEM).

\section{Results and Discussion}

The primary focus here was on the quality of the pattern transfer, the limitations in terms of half-pitch relative to the electrode height and width and the overall constraints put on the process by the overlay mismatch of the e-beam writing.

For the pre-patterning of the insulating layer precise control of the step height between the gate electrode and the source/drain electrode level was essential. Therefore a $2 \mathrm{~nm} \mathrm{Al} \mathrm{O}_{3}$ etch stop was employed. $2 \mathrm{~nm}$ of alumina proved to be enough to withstand over-etching (etch rate of alumina 0.95 $\mathrm{nm} / \mathrm{min}$, silicon nitride: $30.5 \mathrm{~nm} / \mathrm{min}$ ). Slight overetching was necessary in order to compensate for micro- or macro-loading effects [15]

After palladium deposition a smooth step coverage was observed within the transition region, which is of importance in order to be able to spin the HSQ uniformly for the following processing steps.

For the pattern transfer of the HSQ lines into the palladium layer, it was observed that a high degree of profile control could be obtained by adjusting the ratio of palladium to $\mathrm{HSQ}$ thickness and choosing the tilting angle accordingly. The theoretical etch rate of palladium to $\mathrm{HSQ}$ is around $1: 1(\mathrm{Pd}$ etch rate $\sim 20 \mathrm{~nm} / \mathrm{min}$ at $250 \mathrm{~mA}, 10^{\circ}$ tilt $\beta$, see Figure 2) [3]. In reality, an etch rate much closer to $1: 2$ was observed, which is due to the faster propagation of the etch-front in the facets that develop rather than in perpendicular direction to the surface.

The etching of the HSQ hard mask and the palladium follow different trends primarily because their etch products display significantly different levels of redeposition. HSQ is chemically identical to polycrystalline $\mathrm{SiO}_{2}$, with some structural defects and residual $\mathrm{Si}-\mathrm{H}$ bonds. For most materials the sputter yield, whilst neglecting any redeposition, follows a similar trend with a maximum sputter yield at approximately $75^{\circ}$ (relative to the vertical) [16] This is a result of only the re-sputtered atoms close to the surface in the interaction volume being able to escape. Therefore at high tilting angles $(\beta)$ the highest degree of material removal is observed [17]. As the interaction volume mainly depends on the kinetic energy of the ions and the density of the target material, the acceleration voltage is the most important parameter. With decreasing voltage the maximum sputter yield shifts to lower tilting angles, although a significant change can only be observed at very low energies. [16-18]

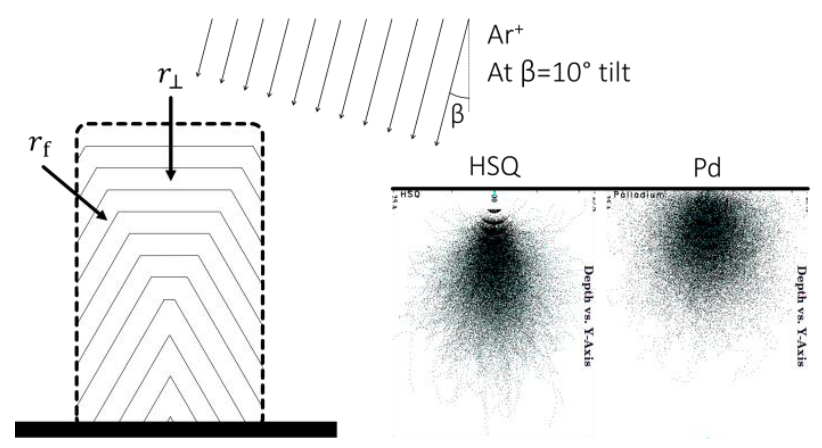

Fig. 2. Facet development due to angle dependent sputter yield $\left(r_{\mathrm{f}}>r_{\perp}\right)$ during etching of HSQ and TRIM simulations of the interaction volume of $500 \mathrm{eV}$ Ar ions with $\mathrm{HSQ}$ and $\mathrm{Pd}$. 
Therefore, when a HSQ line is being ion etched and the corners are not perfectly rectangular, which is in effect always the case, a facet develops very quickly. This facet is therefore finally dominating the resist edge shape (see Figure 2). For amorphous $\mathrm{SiO}_{2}$ layers a maximum sputter yield at tilting angles between $50^{\circ}-60^{\circ}$ has been previously reported [19].

At a $10^{\circ}$ substrate tilt $(\beta)$ during ion milling, a dominant facet with a $29.5^{\circ}$ angle was observed for $\mathrm{HSQ}$ and the calculated etch rate ratio normal to the corresponding surface was $0.36\left(r_{\perp}\right): 0.64\left(r_{\mathrm{f}}\right)$.

Depending on the Pd:HSQ height ratio and the degree of over-etching the HSQ facet can be either completely or not at all transferred into the Pd layer [18]. For a full transfer perfectly triangular electrodes could be observed (see Figure 3). If the facet was not transferred, depending on the tilting angle, trapezoidal to rectangular cross-sections were obtained. All other conditions resulted in polygonal cross-sections. a) $2: 1$

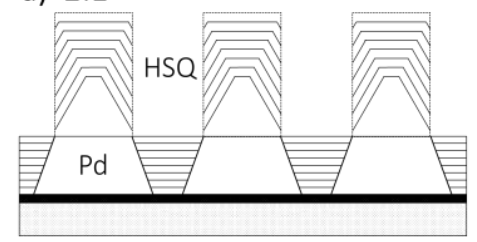

b) $1.75: 1$

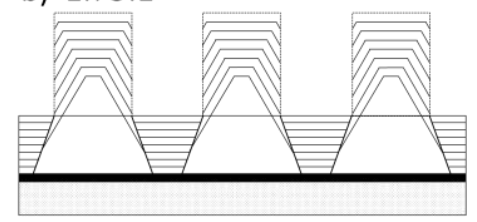

c) $1: 1$

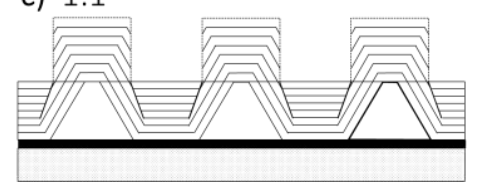

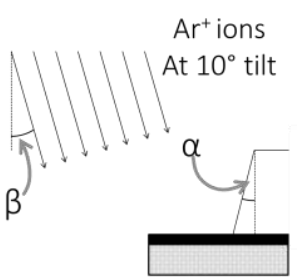
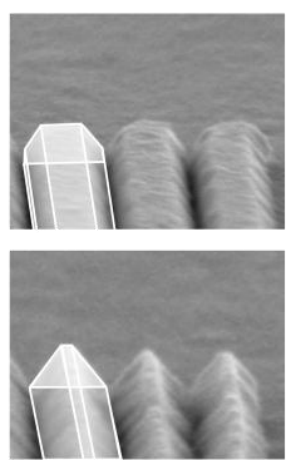

Fig. 3. Different cross-sectional profiles resulting from $\mathrm{Pd}$ : HSQ thickness ratio ranging from 2:1 to $1: 1$.

When no transfer takes place, the sidewall angle (a) of the palladium electrodes can be adjusted between $0^{\circ}$ to $30^{\circ}$ (see Figure 4), as this angle corresponds to the tilt with the maximum sputter yield. A slight broadening of the lines occurs due to an initial crown formation [18]. If the etching is terminated exactly when the facet reaches the $\mathrm{Pd}$ interface no residual crowns are observed after stripping the $\mathrm{HSQ}$. Above $30^{\circ}(\beta)$, with a thick $\mathrm{HSQ}$ layer, the bottoms of the trenches cannot be efficiently sputtered anymore due to excessive shadowing.

Whilst side wall angles ( $\alpha$ ) of up to $30^{\circ}$ could be observed, further flexibility can be obtained by significantly reducing the acceleration voltage. For lower ion energies, the maximum sputter yield is observed at lower angles $(\beta)$ and also more isotropic sputtering is expected, which indicates that the evolving facets in the HSQ layer should propagate much slower [16].

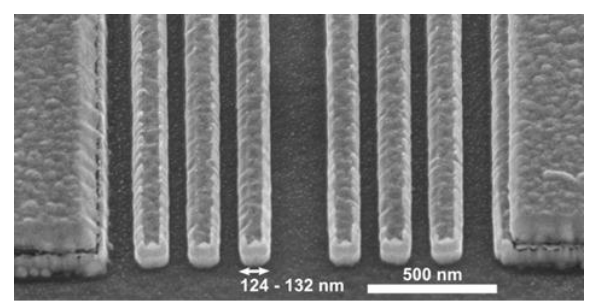

$10^{\circ}: 124-132 \mathrm{~nm}$ width

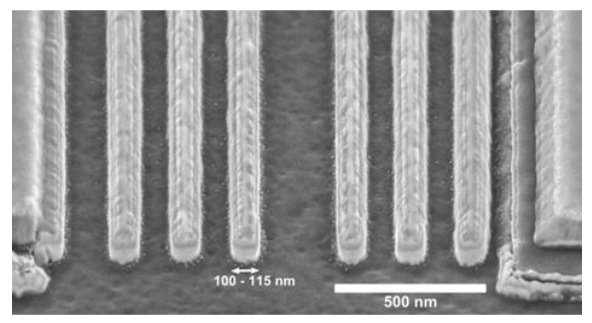

$20^{\circ}: 100-115 \mathrm{~nm}$ width

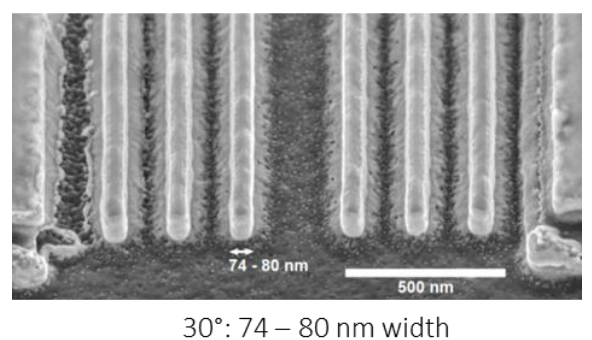

Fig. 4. The result of changing the tilting degree $(\beta)$ from $10^{\circ}$ to $30^{\circ}$. The initial line width was $50 \mathrm{~nm}$, the spacing $150 \mathrm{~nm}$.

Interestingly, for very narrowly spaced electrodes a proximity effect was observed at etching with a $10^{\circ}$ tilt ( $\beta$,see Figure 5$)$. For those lines the side-wall verticality increased with decreasing line spacing. This, most likely, is a result of the locally reduced generation of re-sputtered moieties. A fit with the exposed volume normalized by the total volume between the HSQ lines yields a satisfactory correlation of 0.88 given the small sample size. In other words, electrodes can be spaced much closer than initially anticipated, while still remaining 
spatially separated. At such small distances electrical insulation is not necessarily guaranteed, as very small $P d$ islands may nucleate in areas with low sputter yields. To circumvent this problem, the use of a thicker chromium adhesion layer is suggested. This layer can subsequently be wet etched in order to remove any residual $\mathrm{Cr}$ islands. Above a line spacing of $80 \mathrm{~nm}$ no leakage currents were observed. In this case this corresponds to a line width : line height : half-pitch ratio of $1: 0.6$ : 1.2 (e.g. line width of $120 \mathrm{~nm}$ with a half-pitch of $100 \mathrm{~nm}$ in a $75 \mathrm{~nm}$ palladium layer).

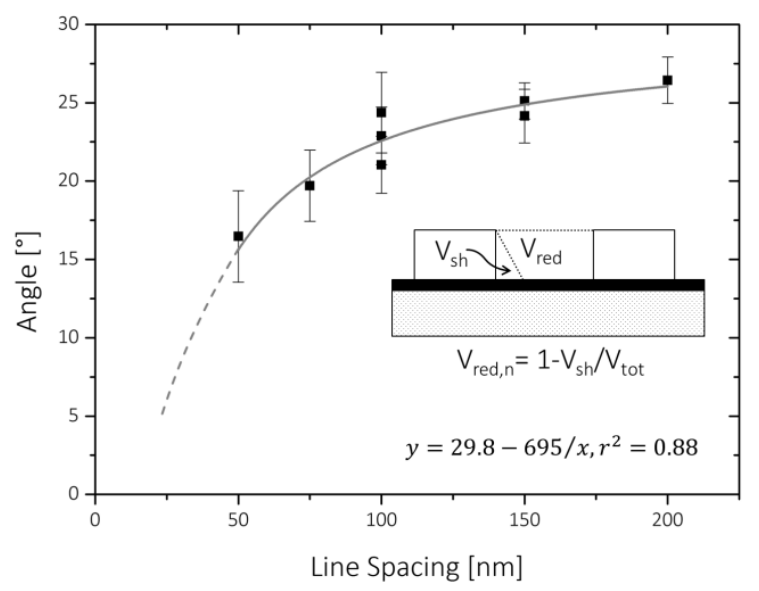

Fig. 5. For smaller spacings, much steeper side-wall angles ( $\alpha$ ) were observed under identical etching conditions at $10^{\circ}$ tilt $(\beta) . V_{s h}$ is the shadowed volume, $V_{\text {tot }}$ the total and $V_{\text {red }}$ the reduced volume. $V_{\text {red,n }}$ is the normalized $\mathrm{V}_{\text {red. }}$

At a given pitch and set footprint, the fabrication of electrodes with a specific side-wall angle $(\alpha)$ can be used to its advantage in order to reduce the parasitic capacitance between adjacent lines as long as the volume reduction is insignificant for the desired application.

When looking at the combination of these two process steps (see Figure 6) it becomes apparent that the process is ultimately not limited by the resolution of the e-beam resists, but rather by the overlay mismatch between the two writing steps. With an e-beam exposure overlay mismatch of less than $15 \mathrm{~nm}$ (as specified by the manufacturer) line spacings of $30 \mathrm{~nm}$ should be feasible without inducing any misalignment.
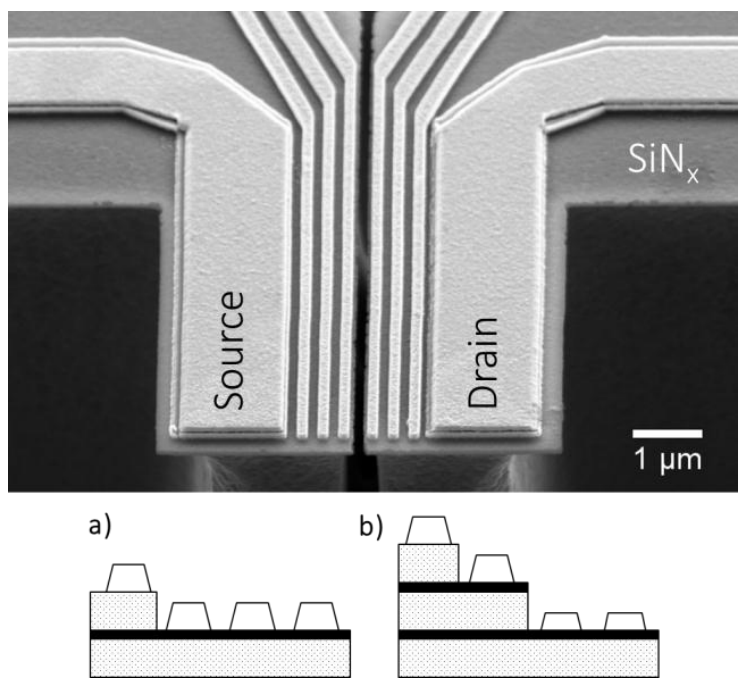

Fig. 6. The end result of the combined process with the cross-section as shown in a). Also more complex profiles with different layer heights such as in b) can be fabricated.

While very thin lines with low line edge roughness can also be fabricated using lift-off, limitations apply in terms of line height and line spacing. By choosing a top-down process in particular, the line spacing can be greatly reduced. Additionally, this process is not only compatible with line-of-sight deposition methods but also with conformal chemical vapor deposition (CVD) methods. One such process of interest here is ALD, as it can yield palladium layers with a very well defined height and low roughness. ALD Pd layers are characterized by a very low percolation threshold of less than $4 \mathrm{~nm}$ [20]. As the minimum pitch to width ratio is expected to scale proportionally with the reduced height, very small features can potentially be fabricated, down to the resolution limit of the ebeam resist. For HSQ it has been demonstrated that lines of $10 \mathrm{~nm}$ can be resolved [21].

Here, a relatively simple topographical structure was desired, but this process is compatible with pre-patterned substrates with a much more complex topography, including multiple levels and materials, as long as the height profile is within the depth of field of the e-beam tool (see Figure 6.b). Direct e-beam writers are characterized by a very high depth of focus of multiple micrometers. Theoretically, layers of different heights can also be patterned simultaneously, while still not inducing any mismatch between the electrodes. Here the alumina layer, which etches much slower than 
palladium ( $2.5 \mathrm{~nm} / \mathrm{min}$ vs. $20 \mathrm{~nm} / \mathrm{min}$ etch rate) can be used to compensate for over-etching.

\section{Conclusions}

We have shown a robust process to fabricate selfaligned electrodes by combining ion milling of palladium with a pre-patterned substrate. Electrodes with a half-pitch of $100 \mathrm{~nm}$ and a height of $75 \mathrm{~nm}$ were successfully fabricated. It was observed that for small resist height the obtained electrode profile is strongly determined by faceting effects, while for thicker resists, the sidewall angle can be adjusted within $0^{\circ}-30^{\circ}$. Additionally, the observed proximity effects should allow patterning of very closely spaced lines, if the other etching parameters are chosen correctly. Under standard e-beam operation, lines with separation as low as $30 \mathrm{~nm}$ can potentially be fabricated without inducing any mismatch.

\section{Acknowledgements}

The authors would like to thank the Swiss National Science Foundation (Project No. 153292) for financially supporting this research. Additionally we would like to acknowledge the co-workers and staff at the Binnig and Rohrer Nanotechnology Center for their support.

\section{References}

[1] Z. Chen, J. Appenzeller, J. Knoch, Y. Lin, P. Avouris, The Role of Metal-Nanotube Contact in the Performance of Carbon Nanotube Field-Effect Transistors, Nano Lett. 5 (2005) 1497-1502. doi:10.1021/nl0508624.

[2] F. Fracassi, Dry etching of palladium thin films in fluorine containing plasmas: X-ray photoelectron spectroscopy investigation, J. Vac. Sci. Technol. A Vacuum, Surfaces, Film. 13 (1995) 63. doi:10.1116/1.579444.

[3] K.R. Williams, K. Gupta, M. Wasilik, Etch rates for micromachining processing-part II, J. Microelectromechanical Syst. 12 (2003) 761-778.

doi:10.1109/JMEMS.2003.820936.

[4] M. Muoth, C. Hierold, Transfer of carbon nanotubes onto microactuators for hysteresis-free transistors at low thermal budget, in: 2012 IEEE 25th Int. Conf. Micro
Electro Mech. Syst., IEEE, 2012: pp. 13521355. doi:10.1109/MEMSYS.2012.6170417.

[5] J. Waissman, M. Honig, S. Pecker, a Benyamini, a Hamo, S. Ilani, Realization of pristine and locally tunable one-dimensional electron systems in carbon nanotubes., Nat. Nanotechnol. 8 (2013) 569-74. doi:10.1038/nnano.2013.143.

[6] C.C. Wu, C.H. Liu, Z. Zhong, One-step direct transfer of pristine single-walled carbon nanotubes for functional nanoelectronics., Nano Lett. 10 (2010) 1032-6. doi:10.1021/nl904260k.

[7] A. Eichler, M. Weiss, S. Oberholzer, C Schönenberger, A. Levy Yeyati, J. Cuevas, et al., Even-Odd Effect in Andreev Transport through a Carbon Nanotube Quantum Dot, Phys. Rev. Lett. 99 (2007) 126602.

doi:10.1103/PhysRevLett.99.126602.

[8] G.A. Steele, G. Gotz, L.P. Kouwenhoven, Tunable few-electron double quantum dots and Klein tunnelling in ultraclean carbon nanotubes., Nat. Nanotechnol. 4 (2009) 363-7. doi:10.1038/nnano.2009.71.

[9] A.W. Barnard, V. Sazonova, A.M. van der Zande, P.L. McEuen, Fluctuation broadening in carbon nanotube resonators. Proc. Natl. Acad. Sci. U. S. A. 109 (2012) 19093-19096.

doi:10.1073/pnas.1216407109.

[10] W.J. Venstra, R. van Leeuwen, H.S.J. van der Zant, Strongly coupled modes in a weakly driven micromechanical resonator, Appl. Phys. Lett. 101 (2012) 243111. doi:10.1063/1.4769182.

[11] T. Longjuan, Z. Yinfang, Y. Jinling, L. Yan, $Z$. Wei, $X$. Jing, et al., Dependence of wet etch rate on deposition, annealing conditions and etchants for PECVD silicon nitride film, J. Semicond. 30 (2009) 096005. doi:10.1088/1674-4926/30/9/096005.

[12] V. V. Afanas'ev, A. Stesmans, B.J. Mrstik, C. Zhao, Impact of annealing-induced compaction on electronic properties of atomic-layer-deposited Al2O3, Appl. Phys. Lett. $81 \quad$ (2002) 1678-1680. doi:10.1063/1.1501163. 
[13] T. Bakke, J. Schmidt, M. Friedrichs, B. Völker, Etch Stop Materials for Release by Vapor HF Etching, MicroMechanics Eur. Work. (2005) 103-106. doi:10.1016/j.diagmicrobio.2010.10.024.

[14] H. Namatsu, Influence of edge roughness in resist patterns on etched patterns, J. Vac. Sci. Technol. B Microelectron. Nanom. Struct. $\quad 16 \quad$ (1998) 3315. doi:10.1116/1.590375.

[15] C. Hedlund, Microloading effect in reactive ion etching, J. Vac. Sci. Technol. A Vacuum, Surfaces, Film. 12 (1994) 1962. doi:10.1116/1.578990.

[16] Q. Wei, K.-D. Li, J. Lian, L. Wang, Angular dependence of sputtering yield of amorphous and polycrystalline materials, J. Phys. D. Appl. Phys. 41 (2008) 172002. doi:10.1088/0022-3727/41/17/172002.

[17] P. Sigmund, Theory of Sputtering., Phys. Rev. 184 (1969) 383-416. doi:http://dx.doi.org/10.1103/PhysRev.184.3 83.

[18] W. Kern, Thin Film Processes II, Elsevier, 1991. http://dx.doi.org/10.1016/B978-0-08052421-4.50019-8 (accessed April 26, 2015).

[19] N. Gosset, M. Boufnichel, E. Bahette, W. Khalfaoui, R. Ljazouli, V. Grimal-Perrigouas, et al., Single and multilayered materials processing by argon ion beam etching: study of ion angle incidence and defect formation, J. Micromechanics Microengineering. $25 \quad$ (2015) 095011. doi:10.1088/0960-1317/25/9/095011.

[20] T. Xu, M.P. Zach, Z.L. Xiao, D. Rosenmann, U. Welp, W.K. Kwok, et al., Self-assembled monolayer-enhanced hydrogen sensing with ultrathin palladium films, Appl. Phys. Lett. 86 (2005) 1-3. doi:10.1063/1.1929075.

[21] A.E. Grigorescu, M.C. van der Krogt, C.W. Hagen, P. Kruit, 10nm lines and spaces written in HSQ, using electron beam lithography, Microelectron. Eng. 84 (2007) 822-824. doi:10.1016/j.mee.2007.01.022. 


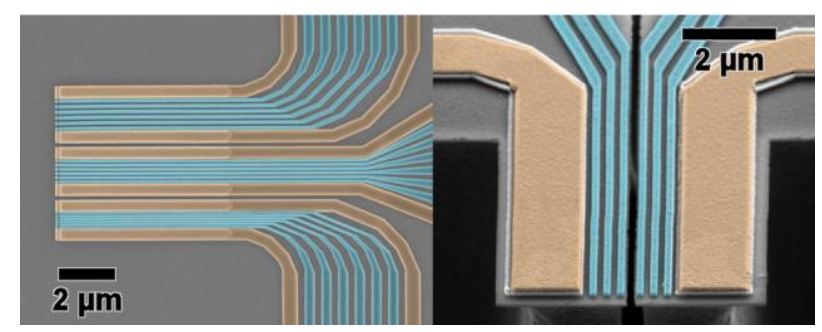

